Applied Mathematical Sciences, Vol. 8, 2014, no. 165, 8287 - 8293

HIKARI Ltd, www.m-hikari.com

http://dx.doi.org/10.12988/ams.2014.411916

\title{
A Buffon - Laplace Type Problems for an Irregular Lattice and with Maximum Probability
}

\author{
D. Barilla, G. Caristi and A. Puglisi \\ Department SEAM, University of Messina \\ Via dei Verid, 75, 98122 Messina, Italy
}

Dedicated to Professor Marius Stoka on the occasion of his $80^{\text {th }}$ birthday

Copyright (c) 2014 D. Barilla, G. Caristi and A. Puglisi. This is an open access article distributed under the Creative Commons Attribution License, which permits unrestricted use, distribution, and reproduction in any medium, provided the original work is properly cited.

\begin{abstract}
In some previous papers [1], [2], [3], [4], [5], [6], [7], [8], [9] and [10] the authors studies same Laplace problems with different fundamental cells. In this paper we consider a lattice with fundamental cell represented in fig. 1 and we compute the probability that a segment of random position and constant length intersects a side of lattice. Then we prove that there are values for parameters that determine the fundamental cell for which the probability determined is maximum.
\end{abstract}

Mathematics Subject Classification: primary: 30C45, 30C80, secondary: 30D

Keywords: Geometric Probability, stochastic geometry, random sets, random convex sets and integral geometry

Let $\Re(a ; b ; \alpha)$ where $\alpha$ is an angle with $\left.\alpha \epsilon] 0, \operatorname{Arctg} \frac{b}{2 a}\right]$ and with the fundamental cell $C_{0}$ represented in fig. 1 


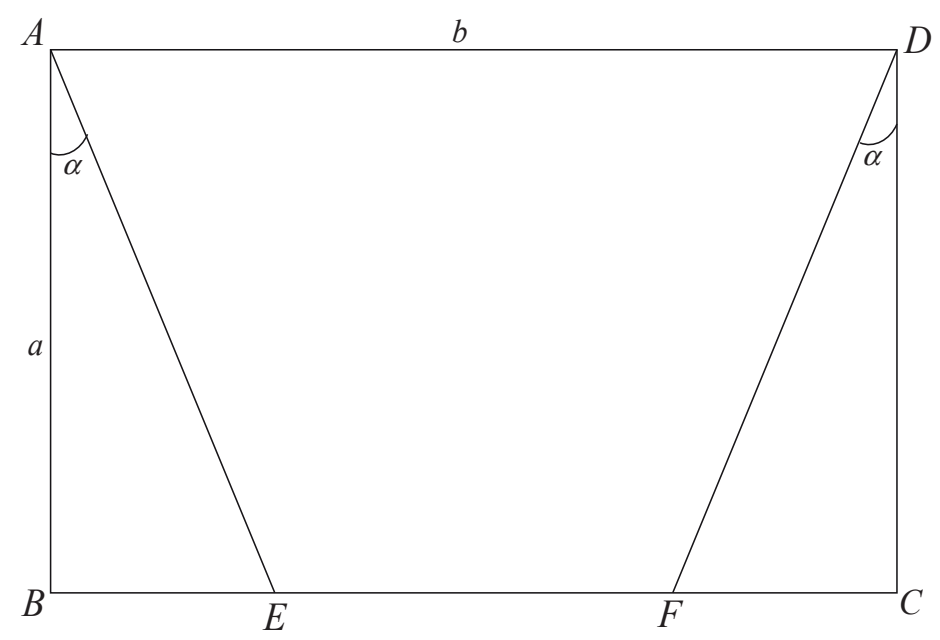

fig.1

By this figure we have

$$
\begin{gathered}
|A E|=|D F|=\frac{a}{\cos \alpha}, \quad|B E|=|C F|=\text { atg } \alpha, \quad|E F|=b-2 a \operatorname{tg} \alpha, \\
\text { area } C_{0}=a b
\end{gathered}
$$

We want to compute the probability that a segment $s$ with random position and of constant length $l<\min ($ atg $\alpha, b-2 a t g \alpha)$ intersects a side of lattice $\Re$, i.e. the probability $P_{\text {int }}$ that the segment $s$ intersects a side of the fundamental cell $C_{0}$.

The position of the segment $s$ is determinated by middle point and by the angle $\varphi$ that $s$ forms with line $B C$ (o $A D)$.

To compute the probability $P_{\text {int }}$ we consider the limit positions of segment $s$, for a fixed value of $\varphi$, in the cells $C_{0 i},(i=1,2,3)$.

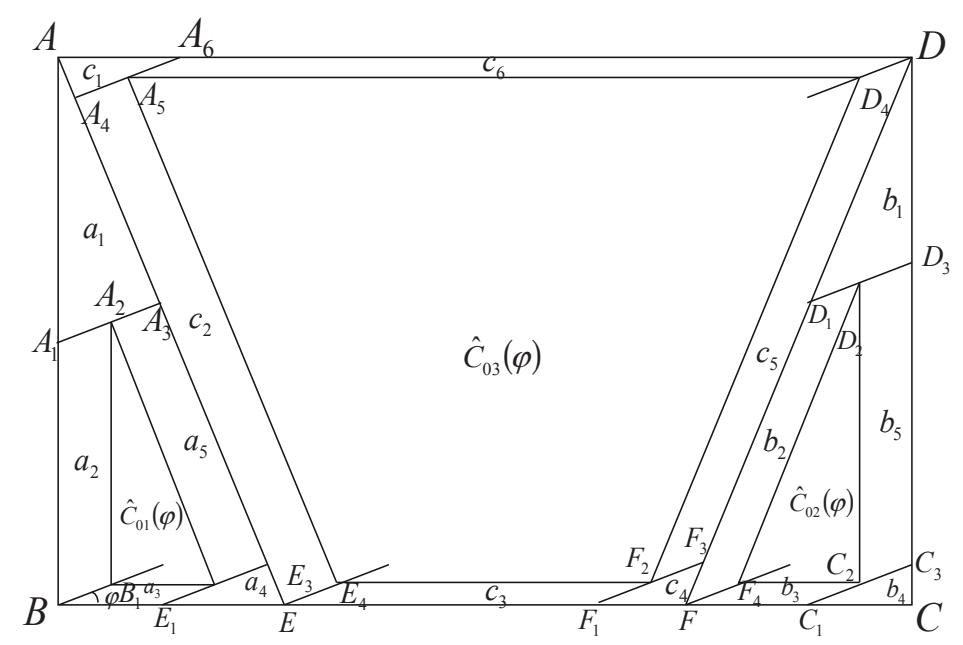

fig. 2 
and the relations

$$
\begin{aligned}
& \operatorname{area} \widehat{C}_{01}(\varphi)=\operatorname{area}_{01}-\sum_{i=1}^{5} \operatorname{areaa}_{i}(\varphi), \\
& \operatorname{area} \widehat{C}_{02}(\varphi)=\operatorname{area}_{02}-\sum_{i=1}^{5} \operatorname{areab}_{i}(\varphi), \\
& \operatorname{area} \widehat{C}_{03}(\varphi)=\operatorname{areaC}_{03}-\sum_{i=1}^{6} \operatorname{areac}_{i}(\varphi) .
\end{aligned}
$$

To compute area $\widehat{C}_{01}(\varphi)$ considering fig.2

$$
\begin{gathered}
\operatorname{areaa}_{1}(\varphi)=\frac{l^{2} \cos \varphi \cos (\varphi-\alpha)}{2 \sin \alpha} . \\
\operatorname{areaa}_{2}(\varphi)=\frac{a l}{2} \cos \varphi-\frac{l^{2} \cos \varphi \cos (\varphi-\alpha)}{2 \sin \alpha} .
\end{gathered}
$$

The relations (7) and (8) give us

$$
\begin{gathered}
\operatorname{areaa}_{1}(\varphi)+\operatorname{area}_{2}(\varphi)=\frac{a l}{2} \cos \varphi . \\
\operatorname{areaa}_{4}(\varphi)=\frac{l^{2} \sin \varphi \cos (\varphi-\alpha)}{2 \cos \alpha} . \\
\operatorname{areaa}_{3}(\varphi)=\frac{a l}{2} \operatorname{tg} \alpha \sin \varphi-\frac{l^{2} \sin \varphi \cos (\varphi-\alpha)}{2 \cos \alpha} . \\
\operatorname{areaa}_{5}(\varphi)=\frac{a l}{2 \cos \alpha} \cos (\varphi-\alpha)- \\
\frac{l^{2}}{2 \sin \alpha} \cos \varphi \cos (\varphi-\alpha)-\frac{l^{2}}{2 \cos \alpha} \sin \varphi \cos (\varphi-\alpha) .
\end{gathered}
$$

By the formulas (5), (6), (7), (8) and (10) we have

$$
\begin{gathered}
A_{1}(\varphi)=\sum_{i=1}^{5} \operatorname{areaa}_{i}(\varphi)=a l(\cos \varphi+\operatorname{tg} \alpha \sin \varphi)-\frac{l^{2}}{4} \\
{[2 \sin 2 \varphi+(\operatorname{ctg} \alpha-\operatorname{tg} \alpha) \cos 2 \varphi+t g \alpha+\operatorname{ctg} \alpha] .}
\end{gathered}
$$

In the same way

$$
\operatorname{areab}_{1}(\varphi)=\frac{l^{2} \cos \varphi \cos (\varphi+\alpha)}{2 \sin \alpha} .
$$




$$
\begin{gathered}
\operatorname{areab}_{2}(\varphi)=\frac{a l}{2 \cos \alpha} \cos (\varphi+\alpha)-\frac{l^{2} \cos \varphi \cos (\varphi+\alpha)}{2 \sin \alpha} . \\
\operatorname{areab}_{4}(\varphi)=\frac{l^{2}}{4} \sin 2 \varphi \\
\operatorname{areab}_{3}(\varphi)=\frac{a l}{2} \operatorname{tg} \alpha \sin \varphi-\frac{l^{2}}{4} \sin 2 \varphi . \\
\operatorname{areab}_{5}(\varphi)=\frac{a l}{2} \cos \varphi-\frac{l^{2}}{4}(1+\cos 2 \varphi) \operatorname{ctg} \alpha .
\end{gathered}
$$

By the relations (12), (13), (14), (15) and (16) follows

$$
A_{2}(\varphi)=\sum_{i=2}^{5} \operatorname{areab}_{i}(\varphi)=\operatorname{al} \cos \varphi-\frac{l^{2}}{4} \operatorname{ctg} \alpha(1+\cos 2 \varphi) .
$$

Now we compute area $\widehat{C}_{03}(\varphi)$.

$$
\begin{gathered}
\operatorname{areac}_{1}(\varphi)=\frac{l^{2} \sin \varphi \cos (\varphi-\alpha)}{2 \cos \alpha} . \\
\operatorname{areac}_{2}(\varphi)=\frac{a l}{2} \cos \varphi-\frac{a l}{2} \operatorname{tg} \alpha \sin \varphi-\frac{l^{2} \sin \varphi \cos (\varphi-\alpha)}{2 \cos \alpha} . \\
\operatorname{areac}_{4}(\varphi)=\frac{l^{2} \sin \varphi \cos (\varphi+\alpha)}{2 \cos \alpha} . \\
\operatorname{areac}_{3}(\varphi)=\frac{(b-2 a t g \alpha) l}{2} \sin \varphi-\frac{l^{2} \sin \varphi \cos (\varphi+\alpha)}{2 \cos \alpha} . \\
\operatorname{areac}_{6}(\varphi)=\frac{b l}{2} \sin \varphi-\frac{l^{2} \sin \varphi \cos (\varphi-\alpha)}{2 \cos \alpha} . \\
\operatorname{areac}_{5}(\varphi)=\frac{a l}{2} \cos \varphi-\frac{a l}{2} \operatorname{tg} \alpha \sin \varphi-\frac{l^{2} \sin \varphi \cos (\varphi+\alpha)}{2 \cos \alpha} .
\end{gathered}
$$

Considering the relations (18), (19), (20), (21), (22) and (23) we can write

$$
A_{3}(\varphi)=\sum_{i=1}^{6} \operatorname{areac}_{i}(\varphi)=\operatorname{alcos} \varphi+\frac{(b-2 a \operatorname{tg} \alpha) l}{2} \sin \varphi-\frac{l^{2}}{4} \sin 2 \varphi
$$

We denote with $M_{i}(i=1,2,3)$ the set of segments $s$ that they have the middle point in the cell $C_{0 i}$ and with $N_{i}$ the set of segments $s$ full content in $C_{0 i}$, we have [12]: 


$$
P_{\text {int }}=1-\frac{\sum_{i=1}^{3} \mu\left(N_{i}\right)}{\sum_{i=1}^{3} \mu\left(M_{i}\right)}
$$

where $\mu$ is the Lebesgue measure in the euclidean plane.

To compute the measure $\mu\left(M_{i}\right)$ and $\mu\left(N_{i}\right)$ we use the kinematic measure of Poincaré [11]:

$$
d k=d x \wedge d y \wedge d \varphi,
$$

where $x, y$ are the coordinate of middle point of $s$ and $\varphi$ the fixed angle $\varphi\left[0, \frac{\pi}{2}\right.$ we have

$$
\begin{gathered}
\mu\left(M_{i}\right)=\int_{0}^{\frac{\pi}{2}-\alpha} d \varphi \iint_{\left\{(x, y) \in C_{0 i}\right\}} d x d y= \\
\int_{0}^{\frac{\pi}{2}-\alpha}\left(\text { area }_{0 i}\right) d \varphi=\left(\frac{\pi}{2}-\alpha\right) \text { area }_{0 i}, \quad(i=1,2,3),
\end{gathered}
$$

then

$$
\sum_{i=1}^{3} \mu\left(M_{i}\right)=\left(\frac{\pi}{2}-\alpha\right) a b
$$

Moreover

$$
\begin{gathered}
\mu\left(N_{i}\right)=\int_{0}^{\frac{\pi}{2}-\alpha} d \varphi \int_{\left\{(x, y) \epsilon \widehat{C}_{0 i}(\varphi)\right\}} d \varkappa d y=\int_{0}^{\frac{\pi}{2}-\alpha}\left[\operatorname{area} C_{0 i}-A_{i}(\varphi)\right] d y= \\
\left(\frac{\pi}{2}-\alpha\right) \text { area } C_{0 i}-\int_{0}^{\frac{\pi}{2}-\alpha}\left[A_{i}(\varphi)\right] d y,
\end{gathered}
$$

then

$$
\sum_{i=1}^{3} \mu\left(N_{i}\right)=\left(\frac{\pi}{2}-\alpha\right) a b-\int_{0}^{\frac{\pi}{2}-\alpha}\left[\sum_{i=1}^{3} A_{i}(\varphi)\right] d \varphi .
$$

The formulas (25), (26), and (27) give us

$$
\begin{gathered}
P_{\text {int }}=\frac{1}{\left(\frac{\pi}{2}-\alpha\right) a b}\left\{\left[3 a \cos \alpha+\frac{b}{2}(1-\sin \alpha)\right] l-\frac{l^{2}}{4}\right. \\
\left.\left[3 \cos 2 \alpha+2+(2 \operatorname{ctg} \alpha+\operatorname{tg} \alpha)\left(\frac{\pi}{2}-\alpha\right)\right]\right\} .
\end{gathered}
$$

Denote 


$$
f(\alpha)=\frac{\left[3 a \cos \alpha+\frac{b}{2}(1-\sin \alpha)\right] l-\frac{l^{2}}{4}\left[3 \cos \alpha+2+(2 \operatorname{ctg} \alpha+\operatorname{tg} \alpha)\left(\frac{\pi}{2}-\alpha\right)\right]}{\frac{\pi}{2}-\alpha},
$$

we have

$$
\begin{gathered}
f^{\prime}(\alpha)=\frac{1}{\frac{\pi}{2}-\alpha}\left\{\left(-3 a \sin \alpha-\frac{b}{2} \cos \alpha\right) l-\frac{l^{2}}{4}[-6 \sin 2 \alpha-2 \operatorname{ctg} \alpha-\operatorname{tg} \alpha+\right. \\
\left.\left.\left(\frac{\pi}{2}-\alpha\right)\left(\frac{1}{\cos ^{2} \alpha}-\frac{2}{\sin ^{2} \alpha}\right)\right]\right\}+\frac{1}{\left(\frac{\pi}{2}-\alpha\right)^{2}}\{[3 a \cos \alpha+ \\
\left.\left.\frac{b}{2}(1-\sin \alpha)\right] l-\frac{l^{2}}{4}\left[3 \cos 2 \alpha+2+(2 \operatorname{ctg} \alpha+\operatorname{tg} \alpha)\left(\frac{\pi}{2}-\alpha\right)\right]\right\} .
\end{gathered}
$$

For

$$
\alpha=\frac{\pi}{4}, \quad b=3 a
$$

and

$$
3\left(2+\sqrt{2}-\frac{\pi \sqrt{2}}{4}\right) a+\left(7-\frac{\pi}{4}\right) l=0
$$

we have

$$
f^{\prime}(\alpha)=0 .
$$

Then, with a simple calculation we show that for these values of parameters $a, b, l, \alpha$ we have

$$
f^{\prime \prime}(\alpha)<0 .
$$

Consequently, for these values the probability $P_{\text {int }}$ is maximum.

\section{References}

[1] D. Barilla, M. Bisaia, E. Saitta, M. Stoka, A Laplace type problems for a lattice with cell composed by two trapezium and a triangle, Applied Mathematical Sciences, Vol. 8, 2014, no. 9, 423 - 439.

[2] D. Barilla, M. Bisaia, G. Caristi and A. Puglisi, On Laplace type problems (I), Journal of Pure and Applied Mathematics: Advances and Applications, vol. 6, n.1, 2011, pp. 51-70. 
[3] D. Barilla, M. Bisaia, G. Caristi and A. Puglisi, On Laplace type problems (II), Far East Journal of Mathematical Sciences, vol. 58, n. 2, 2011, pp. 145-155.

[4] D. Barilla, G. Caristi, A. Puglisi, M. Stoka, A Laplace type problem for two hexagonal lattices of Delone with obstacles, Applied Mathematical Sciences, Vol. 7, no. 92, pp. 4571 - 4581.

[5] D. Barilla, G. Caristi, A. Puglisi, Laplace type for an irregular trapetium lattice and body test rectangle, Applied Mathematical Sciences vol. $7 \mathrm{n}$. 10 pp. $487-501$.

[6] D. Barilla, G. Caristi, E. Saitta, M. Stoka, Laplace type problem for lattice with cell composed by two quadrilaterals and one triangle, Applied Mathematical Sciences, Vol. 8, 2014, no. 16, 789 - 804.

[7] D. Barilla, A. Puglisi, E. Saitta, M. Stoka, A Laplace type problems for a lattice with cell composed by two triangles and a trapezium, Applied Mathematical Sciences, Vol. 8, 2014, no. 9, 441 - 461.

[8] D. Barilla, A. Puglisi, E. Saitta, Laplace type problems for irregular lattice with cell "Pentagon + Triangle", International Mathematical Forum, Vol. 8, no. 23, pp. 1131 - 1142 .

[9] G. Caristi, M. Stoka, A Laplace type problem for Delone Sessadecagonal lattice with obstacles, Mathematical Models and Methods in Applied Sciences Proceedings of the 13th WSEAS International Conference on Mathematics and Computers in Business and Economics (MCBE 12) pp. 124-130.

[10] G. Caristi, M. Stoka, Buffon-Laplace type problems for three regular lattices and "body test" a parallelogram, International Mathematical Forum, vol. 8 n. 6, pp. 245-271.

[11] H. Poincarè, Calcul des probabilitès, 2nd ed., Gauthier-Villard, Paris, 1912.

[12] M. Stoka, Probabilités géométriques de type "Buffon" dans le plan euclidien, Atti Accd. Sci. Torino, T, 110.

Received: September 1, 2014; Published: November 25, 2014 